\title{
Swallowing Function Trial
}

National Cancer Institute

\section{Source}

National Cancer Institute. Swallowing Function Trial. NCI Thesaurus. Code C161478.

A type of trial designed to evaluate the effect of the investigational product on the physiologic act of swallowing. 